\title{
A New Modulus of Smoothness for Uniform Approximation
}

\author{
Eman Samir Bhaya \\ Department of mathematics, college of Education, Babylon University. \\ emanbhaya@itnet.uobabylon.edu.iq \\ Bushra Khudhair Hussein \\ Department of mathematics, college of Education, Babylon University \\ bushrakhudhair999@gmail.com
}

\begin{tabular}{l}
\hline ARTICLE INFO \\
\hline Submission date: $19 / 4 / 2018$ \\
Acceptance date: $14 / 5 / 2018$ \\
Publication date: $14 / 10 / 2018$ \\
\hline
\end{tabular}

\begin{abstract}
The estimates of best approximation using classical modulus of smoothness is not uniform. Also we sometimes need to improve the degree of best approximation near the end points. Thus we need to improve this classical modulus of smoothness. Here we define a new modulus of smoothness to achieve uniform estimates of best approximation and an improvement of a degree of such version of best approximation. Our modulus of smoothness is for k-monotone functions. Estimates for using our modulus of smoothness are introduced. Applications for these estimates are also introduced.
\end{abstract}

Keywords: modulus of smoothness, k-monotone function, best approximation.

\section{Introduction}

Different moduli of smoothness were introduced. But the moduli of smoothness that have the most attention in the recent years are the Ditizian-Totik modulus of smoothness [3], and Ivanov $\tau$ modulus of smoothness [4] For more see [2]. The estimates of best approximation using classical modulus of smoothness is not uniform. Sometimes we need to improve the degree of best approximation near the end points, Hear come the needness of uniform approximation if we want to get uniform estimate then we choose modulus of smoothness other than the ordinary modulus of smoothness. We define several types of moduli of smoothness of kmonotone function in $L_{p}$, spaces for $p<1$. We introduce estimates for these moduli of smoothness and then applications for these estimates. Let $L_{p}[a, b], p<0$ denote the space of $f:[a, b] \rightarrow \mathcal{R}$

$$
\|f\|_{p}=\|f\|_{l_{p[a, b]}}=\left[\int_{a}^{b}|f(x)|^{p} d x\right]^{1 / p}, \quad 0<p<\infty
$$

The $k^{\text {th }}$ divided difference [1] of functions defined on the interval [a, b] is

$$
f\left[x_{0}, x_{1}, \ldots ., x_{k}\right]=\sum_{j=0}^{k} f\left(x_{j}\right) / \omega^{\prime}\left(x_{j}\right),
$$

(C) Journal of University of Babylon for Pure and Applied Sciences (JUBES) by University of Babylon is licensed under a Creative Commons Attribution 4.0 International License 
where

$$
\omega(x)=\prod_{j=0}^{k}\left(x-x_{j}\right) \quad \text { for } k \in\{0\} \cup \mathbb{N} .
$$

The k-monotone function [ 1] defined on the interval $[a, b]$ are those function which the kth divided difference of them are non-negative for any $k+1$ points $x_{0}, x_{1}, \ldots \ldots x_{k}$ in the interval $[a, b]$. If $f \in C^{k}[a, b], f$ is k-monotone on $[a, b]$ iff $f^{(k)}(x) \geq 0$ for $x \in[a, b]$. Where $C^{k}[a, b]$ is the space of all continuous function which $\mathrm{k}$-continuous derivatives.

Let us now define the $\mathrm{k}^{\text {th }}$ order modulus of smoothness for functions $f$ in $l_{p(j)}$, as

$$
\omega_{k}(f, \delta, j)_{p}=\sup _{0<h \leq \delta}\left\|\Delta_{h}^{k}(f, ., j)\right\|_{l_{p(j)}}
$$

where

$$
\Delta_{h}^{k}(f, x, j)=\sum_{i=0}^{k}\left(\begin{array}{l}
k \\
i
\end{array}\right)(-1)^{k-i} f\left(x-\frac{k h}{2}\right), \quad \text { if } x \pm \frac{k h}{2} \epsilon j
$$

Is the $\mathrm{k}^{\text {th }}$ symmetric difference .

\section{The Auxiliary Results}

Here we introduce the results that we need to prove our main theorems.

If $f$ is piecewise k-monotone function, then the following result is true.

\section{Lemma 2.1}

For $f$ is piecewise k-monotone function, $f \in L_{p[a, b]}$ we have

$$
\omega_{1}(f, \delta,[a, b])_{q} \leq c(q, p, s) \delta^{\frac{1}{q}-\frac{1}{p}}\|f\|_{l_{p[a, b]},} \quad 0 \leq q<p \leq 1
$$

Proof

$$
\begin{aligned}
& \omega_{1}(f, \delta,[a, b])_{q}=\sup _{0<h \leq \delta}\left\|\Delta_{h}^{k}(f, ., j)\right\|_{q} \\
& \quad \sup _{|h| \leq \delta}\left\|f\left(x+\frac{h}{2}\right)-f\left(x-\frac{h}{2}\right)\right\| \\
& \leq \delta^{\frac{1}{q}-1}\left(\left\|f\left(x+\frac{h}{2}\right)\right\|_{q}-\left\|f\left(x-\frac{h}{2}\right)\right\|_{q}\right) \\
& \leq \delta^{\frac{1}{q}-1}\left[\delta^{1 / p}\|f\|_{p}\right]
\end{aligned}
$$

$\omega_{1}(f, \delta,[a, b])_{q} \leq c(p, q, s) \delta^{\frac{1}{q}-\frac{1}{p}}\|f\|_{l_{p[a, b]}}$

where $c(p, q, s)$ is appositive constant depends on $p, q$ and $s$

\section{Remark 2.2}

We cannot make the constant $c(p, q, s)$ in (2.1) depending on the set $\mathrm{S}$ since this make (2.1) not valid for any function $f$ in $L_{p}[a, b]$, where $\mathrm{S}$ is the number of changes of k-monotonicity off in $[a, b]$. 


\section{Lemma 2.3}

$$
f_{\beta}(x)=\sin \pi \beta x, \quad 0 \leq x \leq 1
$$

where $\beta \in \mathbb{N}$ we have

$$
\omega_{1}(f, \delta) \leq c(p) \beta \delta
$$

Proof

$$
\begin{aligned}
& \left\|\Delta_{h}^{1}(f, x)\right\|_{p}=\left\|f\left(x+\frac{h}{2}\right)-f\left(x-\frac{h}{2}\right)\right\|_{p} \\
& \quad=\int_{\frac{h}{2}}^{1-\frac{h}{2}}\left|\sin \pi \beta\left(x+\frac{h}{2}\right)-\sin \pi \beta\left(x-\frac{h}{2}\right)\right|^{p} d x \\
& \quad=2^{p}|\sin \pi \beta h / 2|^{p} \int_{\frac{h}{2}}^{1-\frac{h}{2}}|\cos \pi \beta x|^{p} d x \\
& \leq c(p)|\sin \pi \beta h / 2|^{p} \\
& \begin{aligned}
\omega_{1}(f, \delta,[0,1]) \leq & c(p) \sup p_{0<h \leq \delta}|\sin \pi \beta h / 2| \\
\leq & c(p)|\sin \pi \beta \delta / 2| \\
\leq & c(p) \beta \delta
\end{aligned}
\end{aligned}
$$

especially when $\delta=1 / \beta, \beta \rightarrow \infty$ so $\delta^{-\frac{1}{q}+\frac{1}{p}} \omega_{1}\left(f_{\beta}, \delta,[0,1]\right)_{q} \rightarrow \infty$ there for this has no upper bounded with bounded by $\left\|f_{\beta}\right\|_{l_{p[0,1]}}$

\section{Remark 2.4}

$$
\omega_{1}(f, \delta,[a, b])_{q} \leq c(p, q, s) \delta^{\frac{1}{q}-\frac{1}{p}}\|f\|_{l_{p[a, b]}}
$$

We cannot increased the power in (2.1)

Proof

For $p<\infty, \quad f_{\alpha}=x^{-\alpha}, 0<\alpha<\frac{1}{p}$

$f_{\alpha}$ is monotone

$$
\begin{aligned}
& \|f\|_{l_{p[0,1]}}=\left[\int_{0}^{1}|f(x)|^{p} d x\right]^{\frac{1}{p}}=\left[\int_{0}^{1}\left|x^{-\alpha}\right|^{p} d x\right]^{\frac{1}{p}} \\
& =\left[\frac{1}{-\alpha p+1}\right]^{\frac{1}{p}} \\
& \begin{aligned}
\omega_{1}\left(f_{\alpha}, \delta,[0,1]\right)_{q}=\sup \left\|f\left(x+\frac{h}{2}\right)-f\left(x-\frac{h}{2}\right)\right\| \\
=\sup \left\|\left(x+\frac{h}{2}\right)^{-\alpha}-\left(x-\frac{h}{2}\right)^{-\alpha}\right\| \\
\left.\geq \sup _{0<h \leq \delta}(\| x+h / 2)^{-\alpha}\|-\|(x-h / 2)^{-\alpha} \|\right) \\
\geq \sup _{0<h \leq \delta}\left[\int_{0}^{1}\left|\left(x+\frac{h}{2}\right)^{-\alpha}\right|^{q} d x-\int_{0}^{1}\left|\left(x-\frac{h}{2}\right)^{-\alpha}\right|^{q} d x\right]^{\frac{1}{q}} \\
\geq\left[\left(\frac{1+\delta / 2)^{-\alpha q+1}}{-\alpha q+1}-\frac{\left(\frac{\delta}{2}\right)^{-\alpha q+1}}{-\alpha q+1}\right]^{\frac{1}{q}}-\left[\frac{\left(1-\frac{\delta}{2}\right)^{-\alpha q+1}}{-\alpha q+1}-\frac{\frac{(\delta}{2}}{-\alpha q+1}\right]^{\frac{1}{q}}\right.
\end{aligned}
\end{aligned}
$$




\section{Lemma 2.5 [1]}

For $f$ is k-monotone function $L_{p}[-1,1]$ space. so we can find a polynomial $\mathrm{q}_{n}$ with degree not exceeding $n$, satisfies

$$
\left\|f-\mathrm{q}_{n}\right\|_{p} \leq c(p, k) \omega_{k}^{\varphi}\left(f, n^{-1}\right)_{p}
$$

\section{Lemma 2.6 [1]}

If $f \in L_{p}(I), \quad 0<p<1$

$$
E\left(\Delta^{k} L_{p}, \Pi_{n} \cap \Delta^{k}\right)_{p} \leq c(p) n^{-(k+1)} E_{n-k}\left(f^{k}\right) .
$$

\section{Lemma 2.7 [1]}

For $\mathrm{P}_{n}(x) \in \Pi_{n}$ and $0<p<\infty$

$$
E_{n}(f)_{p} \leq c(p) \omega_{r}^{\varphi}\left(f, n^{-1}\right)_{p}
$$

\section{The Main Results}

Here let us introduce our modulus of smoothness and its related results.

\section{Definition 3.1}

for is $f$ k-monotone function and $f \in L_{p}[\mathrm{a}, \mathrm{b}]$ the new measure of smoothness we have

$$
\gamma_{\delta}(f, q, p)=\delta^{q-p}
$$

\section{Remark 3.2}

$$
\gamma_{\delta}(f, q, p)=\delta^{q-p}
$$

We cannot increased the power of $\delta$.

Proof

$$
f_{\epsilon}(x)=(1+x)^{\epsilon-\frac{1}{p}} \quad \epsilon>0, \epsilon-\frac{1}{p} \notin \mathbb{N}_{0}
$$

There $f$ and $-f$ are k-monotone

$$
\begin{aligned}
& \left\|f_{\epsilon}\right\|_{l_{p[0,1]}}=\left[\int_{0}^{1}|f(x)|^{p} d x\right]^{\frac{1}{p}} \\
& =\left[\int_{0}^{1}\left|(1+x)^{\epsilon-\frac{1}{p}}\right|^{p} d x\right]^{\frac{1}{p}} \\
& \geq\left[\frac{1 \epsilon p}{\epsilon p}\right]^{\frac{1}{p}} \geq c(\epsilon, p) \\
& \begin{aligned}
\omega_{1}\left(f_{\epsilon}, \delta,[0,1]\right)_{q}=\sup \left\|f\left(x+\frac{h}{2}\right)-f\left(x-\frac{h}{2}\right)\right\| \\
\quad=\sup \|\left(1+\left(x+\frac{h}{2}\right)^{\epsilon-1 / p}-\left(1+\left(x-\frac{h}{2}\right)^{\epsilon-1 / p} \|\right.\right. \\
\quad \sup _{0<h \leq \delta} \|\left(1+\left(x+\frac{h}{2}\right)^{\epsilon-1 / p}\|-\|\left(1+\left(x-\frac{h}{2}\right)^{\epsilon-1 / p} \|\right.\right. \\
=\sup _{0<h \leq \delta}\left[\int_{0}^{1} \mid\left(1+\left.\left(x+\frac{h}{2}\right)^{\epsilon-\frac{1}{p}}\right|^{q}\right]^{\frac{1}{q}}-\left[\int_{0}^{1} \mid\left(1+\left.\left(x-\frac{h}{2}\right)^{\epsilon-\frac{1}{p}}\right|^{q}\right]^{\frac{1}{q}}\right.\right.
\end{aligned}
\end{aligned}
$$




$$
\begin{aligned}
& \geq \sup _{0<h \leq \delta}\left[\int_{0}^{1} \mid\left(1+\left.\left(x+\frac{h}{2}\right)^{\epsilon-1 / p}\right|^{q}\right]^{\frac{1}{q}} d x-\left[\int_{0}^{1}\left|(1+x)^{\epsilon-1 / p}\right|^{q}\right]^{\frac{1}{q}} d x\right. \\
& =\sup _{0<h \leq \delta}\left[\left[\frac{\left(1+x+\frac{h}{2}\right)^{\epsilon q-\frac{q}{p}+1}}{\epsilon q-\frac{q}{p}+1}\right]_{0}^{1}\right]^{\frac{1}{q}}-\left[\left[\frac{(1+x)^{\epsilon q-\frac{q}{p}+1}}{\epsilon q-\frac{q}{p}+1}\right]_{0}^{1}\right]^{1 / q} \\
& =\sup _{0<h \leq \delta}\left[\frac{\left(2+\frac{h}{2}\right)^{\epsilon-\frac{q}{p}+1}}{\epsilon q-\frac{q}{p}+1}-\frac{\left(1+\frac{h}{2}\right)^{\epsilon q-\frac{q}{p}+1}}{\epsilon q-\frac{q}{p}+1}\right]^{\frac{1}{q}}-\left[\frac{(2)^{\epsilon q-\frac{q}{p}+1}}{\epsilon q-\frac{q}{p}+1}-\frac{(1)^{\epsilon q-\frac{q}{p}+1}}{\epsilon q-\frac{q}{p}+1}\right]^{\frac{1}{q}} \\
& =(1-p) \geq \delta^{q-p}
\end{aligned}
$$

\section{Proposition 3.3}

For $f$ is $\mathrm{k}$-monotone function $L_{p}[-1,1], 0<p<q \leq 1$. then there exist a polynomial $\mathrm{q}_{n}$ of degree not exceeding $n$, satisfies

$$
\left\|f-\mathrm{q}_{n}\right\|_{p} \leq c(p, k) n^{-m} \omega_{k-m}^{\varphi}\left(f, n^{-1}\right)_{p}
$$

Proof

$$
\begin{aligned}
\left\|f-q_{n}\right\|_{p} & \leq c(p, k) \omega_{k}^{\varphi}\left(f, n^{-1}\right)_{p} \\
& \leq c(p, k) n^{-1} \omega_{k-1}^{\varphi}\left(f, n^{-1}\right)_{p} \\
& \leq c(p, k) n^{-2} \omega_{k-2}^{\varphi}\left(f, n^{-1}\right)_{p} \\
& \leq c(p, k) n^{-m} \omega_{k-m}^{\varphi}\left(f, n^{-1}\right)_{p}
\end{aligned}
$$

\section{Theorem 3.4}

Let $0 \leq p<q<1$, and $L_{p}[a, b]$ space. then there exist a polynomial $\mathrm{q}_{n}$ of degree not exceeding $n$, such that

$$
\left\|f-\mathrm{q}_{n}\right\|_{p} \leq c(p, k) n^{-m} \gamma_{\frac{1}{n}}(f, q, p)\left\|f^{(m)}\right\|_{q}
$$

proof

Since $p, q<1,0 \leq m \leq k-1$

then

$$
\begin{aligned}
\omega_{k}^{\varphi}\left(f, n^{-1}\right)_{p} & \leq c(p, k) n^{-m} \omega_{k-m}^{\varphi}\left(f, n^{-1}\right)_{p} \\
& \leq c(p, k) n^{-(q-p)}\|f\|_{p} \\
& \leq c(p, k) n^{-(q-p)}\|f\|_{q}
\end{aligned}
$$

Now, let $0<p<q \leq 1$ and let $f \in L_{p} \cap \Delta^{k}$, where $k$ is chosen such that $f^{(m)} \in L_{p}$ where $0 \leq m \leq k-1$ by using Lemma 2.5 and $f^{(m)} \in \Delta^{k-m}$, we get for any $n \geq$ $k-1$ there exist a polynomial $\mathrm{q}_{n}$ of degree not exceeding $n$, such that

$$
\left\|f-\mathrm{q}_{n}\right\|_{p} \leq c(p, k) n^{-m} \omega_{k}^{\varphi}\left(f, n^{-1}\right)_{p} .
$$

Using Theorem 3.4 we get

$$
\left\|f-\mathrm{q}_{n}\right\|_{p} \leq c(p, k) n^{-m} \gamma_{\frac{1}{n}}(f, q, p)\left\|f^{(m)}\right\|_{q} .
$$


A direct consequence of the above theorem we have

\section{Corollary 3.5}

let $0 \leq p<q<1$ and $f$ is k-monotone function in $L_{p}[a, b]$ space then there is a polynomial $\mathrm{q}_{n}$ of degree not exceeding $n$, with

$$
\left\|f-\mathrm{q}_{n}\right\|_{p} \leq c(p, k) n^{-m-q+p}\left\|f^{(m)}\right\|_{q^{\prime}} \quad 0 \leq m \leq k-1
$$

\section{Theorem 3.6}

For $f \in \Delta^{k}$, and $f$ is k-monotone function in $L_{p}[a, b], p<1$

Such that

$$
f^{(m)} \in L_{p}[a, b], 0 \leq m \leq k-1
$$

we have

$$
\left\|f-\mathrm{q}_{n}\right\|_{p} \leq c\left(p_{1}, p_{2}, p, k\right) \omega_{k-m}\left(f^{(m)}, n^{-1}\right)_{p} n^{-m-q+p}\left\|f^{(m)}\right\|_{p}
$$

Proof

let $\alpha=p(q-1) / q(p-1), 0<p<q \leq 1$. for $f \in \Delta^{k}$ with $f^{(m)} \in L_{p}, 0 \leq m \leq$ $k-1$

$$
\begin{aligned}
\left\|f-\mathrm{q}_{n}\right\|_{p} & =\int_{-1}^{1}\left|f-\mathrm{q}_{n}\right|^{p} d x \\
& \leq c \int_{-1}^{1}\left|f-\mathrm{q}_{n}\right|^{p} d x
\end{aligned}
$$

Choose $0<p_{1}, p_{2}<p \leq 1$, such that

$$
\left\|f-\mathrm{q}_{n}\right\|_{p} \leq c\left(p_{1}, p_{2}\right)\left\|f-\mathrm{q}_{n}\right\|_{p_{1}}\left\|f-\mathrm{q}_{n}\right\|_{p_{2}} .
$$

Then

$$
\begin{aligned}
\left\|f-\mathrm{q}_{n}\right\|_{p} & \leq c\left(p_{1}, p_{2}\right) \int_{-1}^{1}\left|f-\mathrm{q}_{n}\right|^{p_{1}} d x \int_{-1}^{1}\left|f-\mathrm{q}_{n}\right|^{p_{2}} d x \\
& \leq\left(p_{1}, p_{2}\right)\left\|f-\mathrm{q}_{n}\right\|_{p_{1}}^{\alpha}\left\|f-\mathrm{q}_{n}\right\|_{p_{2}}^{1-\alpha}
\end{aligned}
$$

Using (2.2) to obtain $\left\|f-q_{n}\right\|_{p} \leq$

$$
c\left(p_{1}, p_{2}, p, k\right) n^{-m} \omega_{k-m}^{\varphi}\left(f^{(m)}, n^{-1}\right)_{p_{1}}^{\alpha} \omega_{k-m}^{\varphi}\left(f^{(m)}, n^{-1}\right)
$$

Using Corollary 3.5 to get

$$
\left\|f-\mathrm{q}_{n}\right\|_{p} \leq c\left(p_{1}, p_{2}, p, k\right) \omega_{k-m}^{\varphi}\left(f^{(m)}, n^{-1}\right)_{p}^{\alpha} n^{-m-q+p}\left\|f^{(m)}\right\|_{p}
$$

\section{Corollary 3.7}

For $0<p<q \leq 1$ and $0 \leq m \leq k-1$ we have

$$
\left\|f-\mathrm{q}_{n}\right\|_{q}=0\left(n^{-m} l(n)\right)
$$

where

$$
l(n)=n^{-q+p}
$$




\section{Definition 3.8}

Let us denote by $\Delta^{k} L_{p}$ for the set of all k-monotone function $f$ define $(-1,1)$ and satisfying $\|f\| \leq 1$, and by $\Pi_{n}$ the space of all algebraic polynomial of degree not exceeding $n$. Let,

$E\left(\Delta^{k} L_{p}, \Pi_{n}\right)_{p}=\sup _{f \in \Delta^{k} L_{p}} \inf _{\mathrm{q}_{n} \in \Pi_{n}}\left\|f-\mathrm{q}_{n}\right\|_{p}$

Is the degree of best approximation of functions in $\Delta^{k} L_{p}$ a polynomial from $\Pi_{n}$

\section{Theorem 3.9}

For $f \in \Delta^{k} L_{p}$, we have $E\left(\Delta^{k} L_{p}, \Pi_{n}\right)_{p} \leq c(p) n^{-q+p}, \quad 0<p<q \leq 1$.

Proof

Using (3.4) we get

$$
E\left(\Delta^{k} L_{p}, \Pi_{n}\right)_{p} \leq\left\|f-q_{n}\right\|_{p}
$$

Using Corollary 3.5 we get

$$
\begin{aligned}
E\left(\Delta^{k} L_{p}, \Pi_{n}\right)_{p} & \leq c(p, k) n^{-m-q+p}\left\|f^{(m)}\right\|_{q} \\
& \leq c(p, k) n^{p-q}
\end{aligned}
$$

\section{Definition 3.10}

Let $E\left(\Delta^{k} L_{p}, \Pi_{n} \cap \Delta^{k}\right)_{p}=\sup _{f \in \Delta^{k} L_{p}} \inf f_{\mathrm{q}_{n} \in \Pi_{n} \cap \Delta^{k}}\left\|f-\mathrm{q}_{n}\right\|_{p}$

is the degree of best approximation of function from $\Delta^{k} L_{p}$ using k-monotone polynomial in $\Pi_{n}$.

\section{Theorem 3.11}

For $0<p<q \leq 1$ we have $E\left(\Delta^{k} L_{p}, \Pi_{n} \cap \Delta^{k}\right)_{p} \leq c(p, k) n^{p-q}$

Proof

For $\mathrm{m}=0$ in Corollary 3.5 and using Lemma 2.6 and Lemma 2.7

$$
\begin{aligned}
E\left(\Delta^{k} L_{p}, \Pi_{n} \cap \Delta^{k}\right)_{p} & \leq c(p) n^{-(k+1)} E_{n-k}\left(f^{k}\right) \\
& \leq c(p) n^{-(k+1)} \omega_{k}^{\varphi}\left(f^{k}, n^{-1}\right)_{p} \\
& \leq c(p) n^{-(k+1)} n^{p-q}\left\|f^{(k)}\right\|_{p} \\
& \leq c(p) n^{-(k+1)+p-q}
\end{aligned}
$$

\section{Conclusions}

Many works are introduced for the direct theorem approximation using many types of moduli of smoothness, but sometimes we need to improve the degree of best approximation near the endpoints. So we need the uniform approximation. Hear we define a suitable modulus of smoothness to get uniform approximation. 
CONFLICT OF INTERESTS.

There are non-conflicts of interest.

\section{References}

[1] E.S. Bhaya. "On the constrained and unconstrained approximation," Ph.D thesis. University of Baghdad, Iraq, 2003.

[2] E. S. Bhaya and Z. M. Fadd. "Nearly exponential approximation for neural networks," Journal of University of Babylon for Applied Sciences, vol. 26, no.1, pp. 103-106, 2018.

[3] Z. Ditzian and V. Totik. Moduli of smoothness. New York: Springer Series in Computational Mathematics, 1987.

[4] K.G. Ivanov, "Direct and converse theorems for the best algebraic approximation in C $[-1,1]$ and $L_{p}[-1,-1]$ ", Journal of C. R. Acad. Bulgare Sci., vol. 33, no. 10, pp. 1309-1312, 1980.

نظريات التقريب الافضل باستخدام مقياس النعومه الاعتيادي لا تعطي تقريبا منتظمة. كنلك نحتاج في بعض الاحيان لتطوير

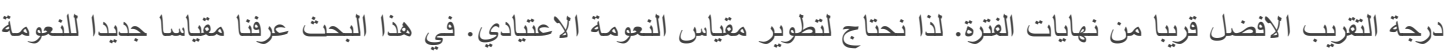

للحصول على تقريبا منتظما وتطوير درجة التقريب الافضل بدلالة هذا المقياس من النعومة. عرفنا هذا المقياس للدوال رتيبة - k .

كذلك قدمنا بعض النظريات المباشرة بدلالة هذا المقياس. بعض تطبيقات هذا المقياس تم تقديمها في هذا البحث ايضا.

الكلمات الادلة: مقياس النعومة , الدالة k- رتيبه, افضل تقريب. 